\title{
Terapéutica del SAHOS (Síndrome de Apnea/Hipopnea Obstructiva del Sueño) desde el punto de vista Ortopédico y Ortodóntico: Revisión de literatura.
}

\section{Therapeutics of OSAHS (Obstructive sleep apnea-hypopnea syndrome (OSAHS) from the Orthopedic and Orthodontic point of view: A literature review.}

\author{
Karina Pazmiño-Rojas ${ }^{1}$ (D) | Christian Yánez-Zurita 2 (D) | Lucitania Goya-Macías ${ }^{3}$ (C)
}

\section{OPEN ACCESS}

Afiliación Institucional

${ }^{1}$ Universidad de Guayaquil, Facultad Piloto de Odontología, Ciencias Básicas, Guayaquil, Ecuador.

${ }^{2}$ Universidad Católica de Cuenca, Facultad de Odontología, Departamento de Ortodoncia, Cuenca, Ecuador.

${ }^{3}$ Universidad de Guayaquil, Facultad Piloto de Odontología, Departamento de Ortodoncia, Guayaquil, Ecuador.

\section{Citaçión:}

Pazmiño-Rojas K, Yánez-Zurita C, GoyaMacías L. Terapia del SAHOS (Síndrome de Apnea/Hipoapnea Obstructiva del Sueño) desde el punto de vista Ortopédico y Ortodóncico: Revisión de literatura. Rev Estomatol. 2021 Noviembre

2021;29(2):e11527. DOI:

10.25100/re.v29i2.11527

Recibido: 12 de Agosto de 2021 Evaluado: 20 de Agosto de 2021 Aceptado: 22 de Octubre de 2021 Publicado: 13 Noviembre de 2021

\section{Correspondencia:}

Karina Pazmiño Rojas. Guayas y 30 de agosto, Santa Rosa, El Oro, Ecuador. Contacto: +59 (39) 96901972 Email: karynapazminorojas@gmail.com

Copyright:

(c) Universidad del Valle.

\begin{abstract}
RESUMEN
Antecedentes: El SAHOS es una afección respiratoria durante el sueño que constituye un conjunto de signos y síntomas causados por la configuración de algunas estructuras anatómicas del sistema estomatognático, derivando en graves alteraciones sistémicas. En los últimos años, las ramas odontológicas de Ortodoncia y Ortopedia han buscado desarrollar alternativas de tratamiento eficaces para mejorar la calidad de vida de estos pacientes.

Objetivo: Describir y resumir las terapias de ortopedia/ortodoncia para el tratamiento de pacientes con el síndrome de apnea/hipopnea obstructiva del sueño (SAHOS).

Materiales y Métodos: Se realizó una búsqueda sistemática en las bases de datos Pubmed, Scielo, Semanthic Scholar y Science Direct de artículos científicos que estudiaron la eficacia de tratamientos ortodóncicos/ortopédicos en pacientes con SAHOS. Se incluyeron estudios en inglés tipo ensayos clínicos controlados publicados en los últimos 5 años.

Resultados: Se encontraron en total 83 artículos, de los cuales solo 9 cumplían con los criterios de elegibilidad. Dentro del tratamiento ortodóncico/ortopédico para el SAHOS, se encontaron dispositivo de avance mandibular, terapia de aparatos orales, terapia posicional; algunos implican efectos adversos en ciertos pacientes

Conclusión: En las terapias de ortodoncia/ortopedia para el tratamiento del SAHOS se observó que los signos y síntomas mejoraron considerablemente a pesar de presentar efectos adversos.
\end{abstract}

\section{PALABRAS CLAVE}

Síndrome de la apnea del sueño; ortodoncia; aparatología ortodóncica; ortopedia maxilar.

\section{ABSTRACT}

Background: OSAHS is a respiratory condition during sleep that constitutes a set of signs and symptoms caused by the configuration of some anatomical structures of the stomatognathic system, leading to serious systemic alterations. In recent years, the dental branches of Orthodontics and Orthopedics have sought to develop effective treatment alternatives to improve the quality of life of these patients.

Objective: To describe and summarize orthopedic/orthodontic therapies for the treatment of patients with obstructive sleep apnea / hypopnea syndrome (OSAHS).

Materials and methods: A systematic search was carried out in the Pubmed, Scielo, Semanthic Scholar and Science Direct databases for scientific articles that studied the efficacy of orthodontic / orthopedic treatments in patients with OSAHS. English-language controlled clinical trials studies published in the last 5 years were included.

Results: A total of 83 articles were found, of which only 9 met the eligibility criteria. Within the orthodontic / orthopedic treatment for OSAHS, mandibular advancement device, oral appliance therapy, positional therapy were found; some involve adverse effects in certain patients.

Conclusion: In the orthodontic / orthopedic therapies for the treatment of OSAHS, it was observed that the signs and symptoms improved considerably despite presenting adverse effects.

KEY WORDS

Sleep apnea syndrome; orthodontics; orthodontic appliances; maxillary orthopedics. 


\section{RELEVANCIA CLÍNICA}

El Síndrome de Apnea-Hipopnea Obstructiva del Sueño (SAHOS) constituye un desorden de prevalencia considerable en la población adulta que involucra a las estructuras del sistema estomatognático obstruyendo el paso del aire, impide la oxigenación adecuada durante el sueño, e implica un factor de riesgo para condiciones sistémicas como hipertensión arterial, accidentes cerebrovasculares o infartos agudos del miocardio, potencialmente mortales para el paciente. En los últimos años, la Ortodoncia y Ortopedia han buscado otorgar una solución a este problema mediante aparatología diversa. El presente trabajo presenta una revisión sistemática de los resultados obtenidos en las terapias de este tipo para tratar el SAHOS.

\section{INTRODUCCIÓN}

El SAHOS es definido como un conjunto de signos y síntomas que se producen durante el sueño provocando la obstrucción del paso del aire debido al colapso de la faringe y que por lo general causan micro despertares con una frecuencia mayor a 5 veces por hora ${ }^{1}$. Las manifestaciones que acompañan a esta condición se han dividido, según el periodo del día, en nocturnos y diurnos. El primer grupo incluye ronquidos, enuresis, sudoración, terrores nocturnos, apneas observadas, sonambulismo, mientras que en el segundo grupo constan respiración bucal, déficit de atención, xerostomía, cefalea matinal ${ }^{1,2}$.

Los factores de riesgo asociados al SAHOS incluyen ser de sexo masculino, la edad media ${ }^{3}$, la obesidad, la retrusión de uno o ambos maxilares, hipertrofia amigdalina ${ }^{4}$, la menopausia, la raza negra, la ingesta de alcohol o depresores del sistema nervioso central, el tabaquismo, base craneal de tamaño disminuido o en posición más vertical de lo normal, maloclusión clase II, macroglosia, posición inferior del hueso hioides, espacio disminuido de la faringe en cualquiera de sus tres porciones o anomalías en el desarrollo craneofacial como el paladar hendido, disostosis o craneosinostosis ${ }^{5}$.

La privación de oxígeno que se produce durante los episodios del SAHOS es responsable de una baja saturación, por lo que existe un compromiso crónico del sueño. Una de las consecuencias que se derivan de esta condición es una mayor probabilidad de sufrir accidentes cardiovasculares ${ }^{6}$. Los patrones que surgen durante las alteraciones nocturnas del síndrome provocan un aumento en la presión arterial sistémica, mayor carga ventricular, arritmias, insuficiencia cardiaca, alteraciones en la circulación pulmonar como hipoxia, hipercapnia y reducción del óxido nítrico alveolar, lo que conlleva a un mayor riesgo de falla cardiopulmonar o cerebrovascular ${ }^{7}$.

La prevalencia del SAHOS es variable, considerando el lugar donde se han desarrollado los estudios. Sin embargo, a nivel mundial se menciona que el $2-3 \%$ de mujeres y el $4 \%$ de hombres adultos padecen esta condición ${ }^{8}$. Otros autores indican que incluso el porcentaje sería hasta del $10 \%$ de población adulta en el mundo?. Las investigaciones coinciden en que existe mayor prevalencia en el sexo masculino ${ }^{10}$, pero que a medida que aumenta la edad, las cifras se equiparan para ambos sexos ${ }^{11}$.
El SAHOS involucra a estructuras que se encuentran dentro del campo de acción de la Odontología, por lo tanto, los profesionales odontólogos se encuentran en una situación privilegiada para valorar, observar y diagnosticar posibles alteraciones del sueño que puedan afectar a los pacientes. La aplicación de tratamientos estomatológicos podría ayudar a reducir las consecuencias derivadas de las afecciones respiratorias durante el sueño ${ }^{12}$.

Si bien la aplicación de presión positiva continua en la vía aérea es un tratamiento de elección en casos de SAHOS severo, también existen otras alternativas que se han propuesto en los últimos años, tales como la cirugía maxilofacial, y el uso de aparatología de ortodoncia/ortopedia para pacientes con cuadros severos o leves. Existen más de 50 dispositivos que tienen esta finalidad, sin embargo, solo un $20 \%$ han sido aprobados por la Food and Drug Administration (FDA). Se han dividido en 4 grupos según su mecanismo de acción: 1. Aparatos de reposición anterior lingual, 2. Aparatos de reposición anterior mandibular, 3. Aparatos de elevación del velo palatino y úvula, y 4. Aparatos de aplicación de presión oral positiva ${ }^{13}$.

El objetivo de este trabajo fue revisar en la literatura científica las terapias de ortopedia/ortodoncia que se practican para tratar pacientes con el síndrome de apnea/hipopnea obstructiva del sueño (SAHOS).

\section{MATERIALES Y MÉTODOS}

\section{Estrategia de búsqueda}

Se llevó a cabo una búsqueda sistemática en las bases de datos Pubmed, Semanthic Scholar, Scielo y Science Direct. Los términos de búsqueda avanzada fueron "Obstructive sleep apnea syndrome" AND "Orthodontic appliance", "Obstructive sleep apnea syndrome" AND "Orthodontic".

\section{Criterios de elegibilidad}

Los criterios de inclusión fueron artículos científicos de tipo ensayo clínico aleatorizado en revistas indexadas, que se hayan publicado entre los años 2017 y 2021. Se excluyeron artículos de otros tipos, de años anteriores a 2017, que estén en diferentes idiomas al inglés y español. Los artículos escogidos debían estar enfocados en la terapia con aparatología ortodóncica/ortopédica para tratar el SAHOS.

\section{RESULTADOS}

Al buscar en las bases de datos acerca del SAHOS y dispositivos de ortodoncia, se obtuvo como resultado 60 artículos, de los cuales solo se eligieron 5 que cumplían con todos los criterios de selección y aportan resultados respecto al tratamiento con algún tipo de dispositivo ortodóncico y ortopédico para el tratamiento de la anomalía en pacientes de diferentes edades.

Cuando se llevó a cabo la búsqueda con los términos SAHOS y 
ortodoncia, se encontró 23 artículos que fueron revisados, luego de lo cual se incluyeron 4 que se enmarcaron en los criterios de inclusión. Entre estos trabajos existieron 2 que indicaban que el tratamiento ortodóncico no obtuvo mejores resultados que otros tipos de terapias para mejorar el cuadro clínico de los pacientes. Por otro lado, 3 investigaciones recomendaron realizar estudios más profundos o con mayor tiempo de evaluación. Los restantes, expresaron que la ortodoncia era una buena alternativa para tratar el síndrome.

\section{DISCUSIÓN}

En la literatura existen numerosos estudios que se han publicado respecto al SAHOS en los últimos años, muchos de los cuales, han comparado los tratamientos tradicionales como la presión positiva continua en las vías aéreas superiores con otras terapias encaminadas hacia la ortodoncia y ortopedia, como el uso de dispositivos durante el sueño, indicando que ya sea por separado $^{14,15}$, o combinadas ${ }^{16}$, presentan muy buenos resultados y es una alternativa adecuada.

Tabla 1. Artículos seleccionados según los criterios de elegibilidad según la búsqueda avanzada de los términos "Obstructive sleep apnea síndrome" AND "Orthodonthic appliance"

\begin{tabular}{|c|c|c|c|c|c|}
\hline $\begin{array}{c}\text { Autor } \\
\text { Año }\end{array}$ & Tipo estudio & Tipo de tratamiento & Duración & Variables & Resultados \\
\hline $\begin{array}{l}\text { Isacsson et al } \\
2017\end{array}$ & ECA & $\begin{array}{l}\text { Aparatos monobloc y } \\
\text { bibloc }\left(\mathrm{Narval}^{\mathrm{TM}}\right)\end{array}$ & 1 año & $\begin{array}{l}\text { IAH } \\
\text { IDO } \\
\text { ESE }\end{array}$ & $\begin{array}{l}\text { Los aparatos monobloc y bibloc son } \\
\text { efectivos al reducir significativamente } \\
\text { los índices analizados }\end{array}$ \\
\hline $\begin{array}{l}\text { Isacsson et al } \\
2019\end{array}$ & $\begin{array}{l}\text { ECA ciego y } \\
\text { equivalencia de } \\
\text { grupos paralelos }\end{array}$ & $\begin{array}{l}\text { Aparatos monobloc y } \\
\text { biblo c }\left(\mathrm{Narval}^{\mathrm{TM}}\right)\end{array}$ & 56 días & $\begin{array}{l}\text { IAH } \\
\text { EA }\end{array}$ & $\begin{array}{l}\text { Los cambios en el IAH fueron }-13,8 \\
\text { para los pacientes con bibloc y }-12,5 \\
\text { para el grupo monobloque. Los eventos } \\
\text { adversos fueron leves y en porcentajes } \\
\text { similares en ambos grupos }\end{array}$ \\
\hline $\begin{array}{l}\text { Godoy et al } \\
2017\end{array}$ & ECA con placebo & DAM & 1,5 años & $\begin{array}{l}\text { ESE } \\
\text { IAH } \\
\text { IAR } \\
\text { PSQI }\end{array}$ & $\begin{array}{l}\text { El IAR, el PSQI y la gravedad de los } \\
\text { síntomas disminuyeron después de } 1,5 \\
\text { años de tratamiento }\end{array}$ \\
\hline $\begin{array}{l}\text { Pitarch et al } \\
2018\end{array}$ & ECA & DAM & 3-6 meses & $\begin{array}{l}\text { IAH } \\
\mathrm{SaO} 2 \mathrm{Min} \\
\mathrm{ES}\end{array}$ & $\begin{array}{l}\text { IAH se redujo de } 22.5 \pm 16.8 \text { a } 9.2 \pm 11.6 \text {. } \\
\text { La } \mathrm{SaO} 2 \text { min mejoró significativamente } \\
\text { al igual que ES }\end{array}$ \\
\hline
\end{tabular}

ECA= Ensayo clínico aleatorizado; IAH= Índice apnea-hipopnea; IDO= Índice de desaturación de oxígeno; ESE= Escala de somnolencia Epworth; EA= Eventos adversos; DAM= Dispositivo de avance mandibular; IAR= Índice de alteración respiratoria; PSQI= Índice de calidad de sueño de Pittsburgh; TAO= Terapia de aparatos orales; $\mathrm{TP}=$ Terapia posicional; $\mathrm{AME}=$ Alivio medio de la enfermedad; $\mathrm{CV}=\mathrm{Calidad}$ de vida; $\mathrm{SaO} 2 \mathrm{~min}=$ Saturación mínima de oxígeno; $\mathrm{ES}=$ Eficiencia de sueño;

Estos resultados difieren de los obtenidos en un ensayo clínico cuyo objetivo fue evaluar la adherencia de la presión positiva continua de las vías aéreas superiores después de usar durante un mes una mascarilla nasal con un dispositivo de avance mandibular, y compararla con la adherencia producida por una mascarilla oronasal. Como resultado obtuvieron que la terapia combinada generaba molestias a los participantes, por lo cual concluyeron que, debido a esta variable, es posible que sean pocos los pacientes que estén dispuestos a seguir este tratamiento ${ }^{17}$.
También existe una investigación en la que seleccionaron a 64 pacientes con alteraciones respiratorias durante el sueño leve y moderada, y se les asignaron aleatoriamente un dispositivo de avance mandibular, presión positiva continua en las vías aéreas superiores o un placebo intraoral titulado. A pesar de que los sujetos del estudio mostraron mejorías en cuanto a su condición, no existieron diferencias significativas entre uno u otro método ${ }^{18}$.

Por otro lado, se comparó la efectividad, así como posibles efectos colaterales, de dos aparatosde avance mandibular, uno ti- 
Pazmiño-Rojas y cols.

Tabla 2. Artículos seleccionados según los criterios de elegibilidad según la búsqueda avanzada de los términos "Obstructive sleep apnea síndrome" AND "Orthodonthic"

\begin{tabular}{|c|c|c|c|c|c|}
\hline $\begin{array}{c}\text { Autor } \\
\text { Año }\end{array}$ & Tipo estudio & Tipo de tratamiento & Duración & Variables & Resultados \\
\hline $\begin{array}{l}\text { Uniken et al } \\
2020\end{array}$ & ECA & DAM & 10 años & $\begin{array}{l}\text { IAH } \\
\mathrm{SaO} 2 \mathrm{~min}\end{array}$ & $\begin{array}{l}\text { El IAH se modificó de } 31.7 \pm 20.6 \text { a } \\
9.9 \pm 10.3 \text {. La SaO2min pasó de } \\
79.6 \pm 6.8 \text { a } 85.3 \pm 5.0 \text {. La ES se modificó } \\
\text { de } 86.6 \pm 12.3 \text { a } 89.4 \pm 11,9 \text {. Las HS } \\
\text { pasaron de } 7: 38 \pm 1: 35 \text { a } 6: 56 \pm 0: 54 \text {. Los } \\
\text { resultados mostraron mejorías } \\
\text { significativas }(P<.05)\end{array}$ \\
\hline $\begin{array}{l}\text { Banhiran et al } \\
2018\end{array}$ & ECA & $\begin{array}{l}\text { Férula termoplástica } \\
\text { ajustable de avance } \\
\text { mandibular }\end{array}$ & 6 semanas & IAH & $\begin{array}{l}\text { Los cambios en el IAH fueron desde } \\
39.2 \pm 2.53 \text { a } 12.92 \pm 2.05 \text { después de la } \\
\text { intervención }\end{array}$ \\
\hline $\begin{array}{l}\text { De Vries et al } \\
2019\end{array}$ & $\begin{array}{l}\text { ECA } \\
\text { multicéntrico }\end{array}$ & DAM & 12 meses & $\begin{array}{l}\text { IAH } \\
\mathrm{CS}\end{array}$ & $\begin{array}{l}\text { El IAH se redujo después de los } 12 \\
\text { meses. Los CS fueron más elevados con } \\
\text { el DAM }\end{array}$ \\
\hline
\end{tabular}

$\mathrm{ECA}=$ Ensayo clínico aleatorizado; $\mathrm{IAH}=$ Índice apnea-hipopnea; DAM= Dispositivo de avance mandibular; SaO2min= Saturación mínima de oxígeno; $\mathrm{ES}=$ Eficiencia de sueño; $\mathrm{CS}=$ Costes sociales

tulable, en pacientes con cuadros de dificultad respiratoria moderada durante el sueño. Después de utilizar ambos dispositivos, se observó que los signos y síntomas mejoraron considerablemente, por lo que se estableció su efectividad para este tipo de anomalías ${ }^{19}$.

Otra investigación comparó aparatos monobloque y bibloque para tratar el SAHOS en 302 pacientes, llegando a la conclusión de que su uso es positivo como tratamiento alternativo de la patología. Sin embargo, la evaluación se realizó después de 56 días, por lo tanto, los autores sugirieron que era necesario llevar estudios similares en un mayor periodo de tiempo para profundizar tanto en los beneficios como en los posibles daños causados por los $\operatorname{dispositivos}^{20}$.

Es así como se publica al año siguiente un estudio que comparó los mismos dispositivos utilizados en el mismo tamaño de muestra durante un año. Después de este periodo de tiempo, fue concluyente que ambos aparatos lograron muy buenos resultados disminuyendo drásticamente las alteraciones respiratorias durante el sueño. Sin embargo, los autores declararon que varios participantes que utilizaron el bibloque abandonaron el ensayo ${ }^{21}$.

Un ensayo clínico controlado aleatorizado tuvo como objetivo comparar la eficacia de un dispositivo de reposicionamiento mandibular hecho a medida y uno prefabricado en un grupo de 25 pacientes con SAHOS leve durante 3 meses. Al cabo de este periodo de tiempo, los autores notaron diferencias significativas en los efectos de ambos aparatos, ya que el que estaba hecho a la medida eliminó algunos signos como la somnolencia diurna excesiva. Como conclusión, se determinó que solo el dispositivo de reposicionamiento mandibular hecho a medida era efectivo en el tratamiento, mientras que el prefabricado no tenía un efecto positivo ${ }^{22}$.

Otra de las terapias que se han comparado a la presión positiva continua para tratar el SAHOS, son los aparatos bucales termoplásticos de avance mandibular mediante un ensayo controlado aleatorio, en el cual se incluyeron a 50 pacientes, 7 de los cuales se retiraron por diferentes motivos. Luego de la intervención, se pudo determinar que ambos métodos tenían efectos indeseables. Sin embargo, los dos tratamientos mejoraron la semiología de los participantes, siendo más eficaz la presión positiva continua ya que llevó los parámetros medidos más cerca de lo normal. Los autores concluyeron que los aparatos bucales termoplásticos pueden ser solo una alternativa temporal para el manejo del síndrome ${ }^{23}$.

Los aparatos orales empleados en el tratamiento de obstrucciones de vías áreas superiores durante el periodo de sueño pueden ser beneficiosos ya que disminuyen los signos y síntomas en los pacientes, pero puede existir variabilidad en cuanto a su efectividad. Se ha sugerido que es posible predecir la efectividad de un dispositivo según el compromiso anatómico y el grado de colapso del tracto respiratorio involucrado ${ }^{24}$. 


\section{CONCLUSIÓN}

Luego de evaluar los resultados del presente trabajo, se concluye que en la literatura científica se han expuesto diversas terapias de ortodoncia/ortopedia que han demostrado ser eficaces en el tratamiento de pacientes que presentan el síndrome apnea/hipopnea obstructiva del sueño (SAHOS).

\section{DECLARACIÓN DE CONFLICTO DE INTERÉS}

Los autores declaran no tener ningún conflicto de interés.

\section{FUENTES DE FINANCIACIÓN}

El presente trabajo no tuvo financiación.

\section{REFERENCIAS}

1. Nogueira F, Borsini E, Cambursano H, Smurra M, Dibur E, Franceschini C, et al. Guías Prácticas de diagnóstico y tratamiento del síndrome de apneas e hipopneas obstructivas del sueño: Actualización 2019. Revista Americana de Medicina Respiratoria. 2019; 19(1): p. 59-90.

2. Ringler F, Gajardo P. Síndrome de apnea obstructiva del sueño persistente en niños adenoamigdalectomizados: artículo de revisión. Rev. Otorrinolaringol. Cir. Cabeza Cuello. 2021; 81: p. 139-152.

3. García M, Cuspineda E, Valiente C. Síndrome de apnea hipopnea del sueño: rol protagónico del ortodoncista. Rev. Haban. Cienc. Méd. 2014; 13(2): p. 207-218.

4. Araoz R, Virhuez Y, Guzmán H. Síndrome de Apnea Obstructiva del Sueño como factor de riesgo para otras enfermedades. Rev. Cient. Cienc. Méd. 2011; 14(1): p. 25-30.

5. Páez-Moya S, Vega-Osorio P. Factores de riesgo y asociados al síndrome de apnea-hipopnea obstructiva del sueño (SAHOS). Rev. Fac. Med. 2017; 65(1): p. 21-24.

6. Ceolim M, Silva C. Alto riesgo para el síndrome de apnea obstructiva del sueño en pacientes con infarto agudo del miocardio. Rev. Latino-Am. Enfermagem. 2015; 23(5): p. 797-805.

7. Morales-Blanhir J, Valencia-Flores M, Lozano-Cruz O. El síndrome de apnea obstructiva del sueño como factor de riesgo para enfermedades cardiovasculares y su asociación con hipertensión pulmonar. Neumol Cir Torax. 2017; 76(1): p. 51-60.

8. Carrillo J, Arredondo F, Reyes M, Castorena A, Vásquez J, Torre L. Síndrome de apnea obstructiva del sueño en población adulta. Neumol Cir Torax. 2010; 69(2): p. 103-115.

9. Kong D, Qin Z, Wang W, Kang J. Effect of obstructive sleep apnea on carotid artery intima media thickness related to inflammation. Clin Invest Med. 2017; 40(1): p. 25-33.

10. Tapia L, Ricardo M, Fernández P, Rabino A. Estudio retrospectivo: características epidemiológicas de los pacientes con síndrome de apnea e hipoapnea obstructiva del sueño y roncopatía intervenidos por faringoplastia, Hospital Británico de Buenos Aires, 2011-2016. Medicina U.P.B. 2019; 38(1): p. 11-16.

11. Hidalgo E, Fuchslocher G, Vargas M, José P. Orthodontist's role in the treatment of snoring and sleep apnea. Revista Médica Clínica Las Condes. 2013; 24(3): p. 501-509.

12. Sharma S, Essick G, Schwartz D, Aronsky A. Sleep Medicine Care Under One Roof: A Proposed Model for Integrating Dentistry and Medicine. J Clin Sleep Med. 2013; 9(8): p. 827-833.

13. Barceló A, Soler M, Riutord P. Odontología y síndrome de apneas-hipopneas del sueño. Evidencias y necesidades para su integración. Medicina Balear. 2017; 32(2): p. 23-29.
14. Uniken J, Doff M, Joffe-Sokolova D, Wijkstra P, Van der Hoeven J, Stegenga B, et al. Long-term obstructive sleep apnea therapy: a 10-year follow-up of mandibular advancement device and continuous positive airway pressure. J Clin Sleep Med. 2020; 16(3): p. 353-359.

15. Tong B, Tran C, Ricciardiello A, Donegan M, Chiang A, Szollosi I, et al. CPAP combined with oral appliance therapy reduces CPAP requirements and pharyngeal pressure swings in obstructive sleep apnea. J Appl Physiol. 2020; 129(5): p. 1085-1091.

16. De Vries G, Hoekema A, Vermeulen K, Claessen J, Jacobs W, Van der Maten J, et al. Clinical- and Cost-Effectiveness of a Mandibular Advancement Device Versus Continuous Positive Airway Pressure in Moderate Obstructive Sleep Apnea. J Clin Sleep Med. 2019; 15(10): p. 1477-1485.

17. Léotard A, Lesgoirres M, Daabek N, Lebret M, Bailly S, Verain A, et al. Adherence to CPAP with a nasal mask combined with mandibular advancement device versus an oronasal mask: a randomized crossover trial. Sleep Breath. 2019; 23(3): p. 885-888.

18. Aarab G, Nikolopoulou M, Ahlberg J, Heymans M, Hamburguesa H, De Lange $\mathrm{J}$, et al. Oral appliance therapy versus nasal continuous positive airway pressure in obstructive sleep apnea: a randomized, placebo-controlled trial on psychological distress. Clin Oral Investig. 2017; 21(7): p. 2371-2378.

19. Al-Dharrab A. A randomized cross over study comparing the efficacy of two mandibular advancement appliances in the treatment of mild-moderate obstructive sleep apnea. Cranio. 2017; 35(6): p. 379-384.

20. Isacsson G, Nohlert E, Fransson A, Bornefalk-Hermansson A, Wiman E, Ortlieb E, et al. Use of bibloc and monobloc oral appliances in obstructive sleep apnoea: a multicentre, randomized, blinded, parallel-group equivalence trial. Eur J Orthod. 2019; 41(1): p. 80-88

21. Tegelberg A, Nohlert E, Bornefalk-Hermansson A, Fransson A, Isacsson G. Respiratory outcomes after a 1-year treatment of obstructive sleep apnoea with bibloc versus monobloc oral appliances: a multicentre, randomized equivalence trial. Acta Odontol Scand. 2020; 78(6): p. 401-408.

22. Johal A, Haria P, Manek S, De Pascua V, Riha R. Ready-Made Versus CustomMade Mandibular Repositioning Devices in Sleep Apnea: A Randomized Clinical Trial. J Clin Sleep Med. 2017; 13(2): p. 175-182.

23. Banhiran D, Assanasen P, Nopmaneejumrudlers C, Nujchanart N, Srechareon $\mathrm{W}$, Chongkolwatana $\mathrm{C}$, et al. Adjustable thermoplastic oral appliance versus positive airway pressure for obstructive sleep apnea. Laryngoscope. 2018; 128(2): p. 516-522.

24. Edwards B, Andara C, Landry S, Sands S, Joosten S, Owens R, et al. UpperAirway Collapsibility and Loop Gain Predict the Response to Oral Appliance Therapy in Patients with Obstructive Sleep Apnea. Am J Respir Crit Care Med. 2016; 194(11): p. 1413-1422. 